\title{
Using biomarkers and early prophylactic treatment to prevent cardiotoxicity in cancer patients on chemotherapy
}

\author{
Giulia Bacchiani" and Daniela Cardinale\# \\ ${ }^{*}$ Cardiology Division, European Institute of Oncology, I.R.C.C.S., \\ Milan, Italy \\ \#Cardioncology Unit, European Institute of Oncology, I.R.C.C.S., \\ Milan, Italy

\section{Address for correspondence:} \\ Daniela Cardinale, \\ Cardioncology Unit \\ European Institute of Oncology \\ Via Ripamonti 435 \\ 2014I Milan \\ Italy
}

Email: daniela.cardinale@ieo.it

\section{INTRODUCTION}

Anticancer therapy can frequently result in serious side effects on specific organs and cardiac toxicity is of considerable concern. Once toxicity develops it may compromise the treatment's clinical effectiveness independent of the oncologic prognosis.( ${ }^{(1)}$ The most frequent - and feared - clinical manifestation of cardiotoxicity is the development of left ventricular dysfunction (LVD), that may impact on the patient's survival and quality of life; thus the gain in life expectancy due to anticancer therapy might be compromised by the risk of increased cardiac mortality. Moreover - even when asymptomatic - LVD not only negatively influences patients cardiologic outcomes - it also seriously limits their therapeutic opportunities when adjunctive oncologic therapy for cancer relapse or persistence is required. The presence of LVD restricts the choice of possible oncologic treatments to the less aggressive and, consequently, less effective options.

Over the last years, the weight of the cardiac toxicity of anticancer treatment has markedly increased due to an improvement in patient survival; an ageing population; the tendency to use progressively higher doses of anthracyclines; the introduction of new anticancer drugs with unexpected cardiac toxicity; and combined treatments with synergistic harmful effects on the heart. (2,3) According to American cardiologic guidelines, patients receiving anticancer treatment may at present be considered a Stage A heart failure group, namely those with an increased risk of developing LVD. ${ }^{(4)}$ Avoiding cardiotoxicity has therefore become a primary

\section{ABSTRACT}

Cardiac toxicity induced by anticancer therapy is of considerable concern for, once it develops, it may compromise the clinical effectiveness of treatment independent of the oncologic prognosis. The main strategy to minimize cardiotoxicity is to detect high-risk patients and begin prophylactic treatment as early as possible. According to the current standard for monitoring cardiac function cardiotoxicity is usually detected only once a functional impairment has already occurred, thus precluding any chance of prevention. The measurement of cardio-specific biomarkers can be a valid diagnostic tool for the early identification, assessment and monitoring of cardiotoxicity.

The role of Troponin I in identifying patients with subclinical cardiotoxicity and their subsequent treatment with angiotensin-converting enzyme inhibitors to prevent left ventricular ejection fraction (LVEF) reduction and cardiac events, is emerging as an effective strategy against these complications. When this approach is not feasible, a complete LVEF recovery and a reduction in cardiac events may be achieved if left ventricular dysfunction (LVD) is detected early and the patient promptly treated with angiotensin-converting enzyme inhibitors, possibly in combination with beta-blocking agents. SAHeart 2012; 9:250-262

goal for both cardiologists and oncologists. The main strategy to prevent clinical cardiotoxicity is early detection and the initiation of prompt prophylactic therapy.

\section{EARLY DETECTION OF CARDIOTOXICITY Current approach}

Periodic assessment for LVEF is the most widely used non-invasive method for detecting cardiotoxicity LVEF either via echocardiography or multigated acquisition scanning. Although several available guidelines exist none specify how often, by what means or how long cardiac function should be monitored during and after cancer treatment. The recommendations of these guidelines are not consistent and seem to be based on modest evidence. ${ }^{(5)}$

Though LVEF is a crude index of cardiac function, it is relatively insensitive in detecting anticancer drug-induced cardiotoxicity at an early stage. Usually there are compensatory mechanisms in place to ensure normal overall function in a patient despite an initial reduction in myocardial contractility. As a rule no considerable 
change in LVEF occurs before the compensatory mechanisms are exhausted.

Once LVD develops, complete recovery of cardiac function occurs in only $42 \%$ of patients, despite optimal pharmacologic therapy. ${ }^{(6)}$ The diagnosis of cardiotoxicity evidenced by a decrease in LVEF therefore precludes any chance of preventing its development. . $^{(5,-9)}$ Measuring LVEF also presents a number of challenges with regards to image quality, assumption of left ventricular geometry, load dependency and expertise. ${ }^{(10)}$ Improved accuracy and reproducibility of LVEF measurements is an actual need in patients receiving anticancer therapy, since clinical decision making relies completely on these measurements. Multiple gated acquisition (MUGA) scan can reduce inter-observer variability with the disadvantages of including the exposure to radioactivity and limited information that can be obtained on cardiac structure and diastolic function. Magnetic resonance imaging (MRI) is considered the gold standard for the evaluation of cardiac volumes, mass, and both systolic and diastolic function. ${ }^{(5)}$ However, high cost and the lack of availability limit the routine use of MRI.

These limitations have prompted investigations into the development of more sophisticated echocardiographic techniques, like contrast echocardiography and real-time 3D-echocardiography, which allow for an improvement in the accuracy of calculating LVEF. $(10,11)$ Both Doppler tissue imaging and speckle-tracking echocardiography appear to be more sensitive than standard echocardiography in detecting subclinical changes in cardiac performance that anticipate a decrease in conventional LVEF, even if long-term data on large populations, confirming the clinical relevance of such changes, are not available yet. ${ }^{(10,1)}$

\section{The role of biomarkers}

A novel approach based on the use of cardiac biomarkers has emerged in the last decade, resulting in a cost-effective diagnostic tool for early, real time identification, assessment and monitoring of anticancer drug-induced cardiotoxicity. This approach seems to overcome most of the limitations of the techniques described above, as it has proven to be more sensitive and more specific, cheaper, repeatable without damage for the patients, readily available even in small hospitals, and without inter-observer variability.

\section{Troponins}

Troponin $(T n)$ is considered the gold standard biomarker for myocardial injury from any cause. ${ }^{(12)}$ The employment of cardiac troponins as biomarkers of early chemotherapy-induced cardiotoxicity was initially reported in animal models.(13) Moving to the clinical field, Lipshultz, et al. ${ }^{(14)}$ reported that, in children treated with anthracyclines (AC) for lymphoblastic leukaemia, a TnT increase in about 30\% of cases, sometimes persisting for months, suggesting that cardiac damage, elicited by AC, may last for a long time. This increase positively correlated with $A C$ dose and predicted morbidity and mortality.

More recently, in the same population, followed-up for 5 years after treatment, the authors observed that children who had experienced at least one raise in $\mathrm{TnT}$ during treatment showed late cardiac abnormalities at echocardiography. ${ }^{(15)}$ Further studies have demonstrated that $\mathrm{Tnl}$ is also a sensitive and specific marker for myocardial injury in adults treated with high-dose chemotherapy (CT), and is able to predict, at a very early phase, both development and severity of future LVD (Figure I). ${ }^{(16-18)}$ Additionally, in 703

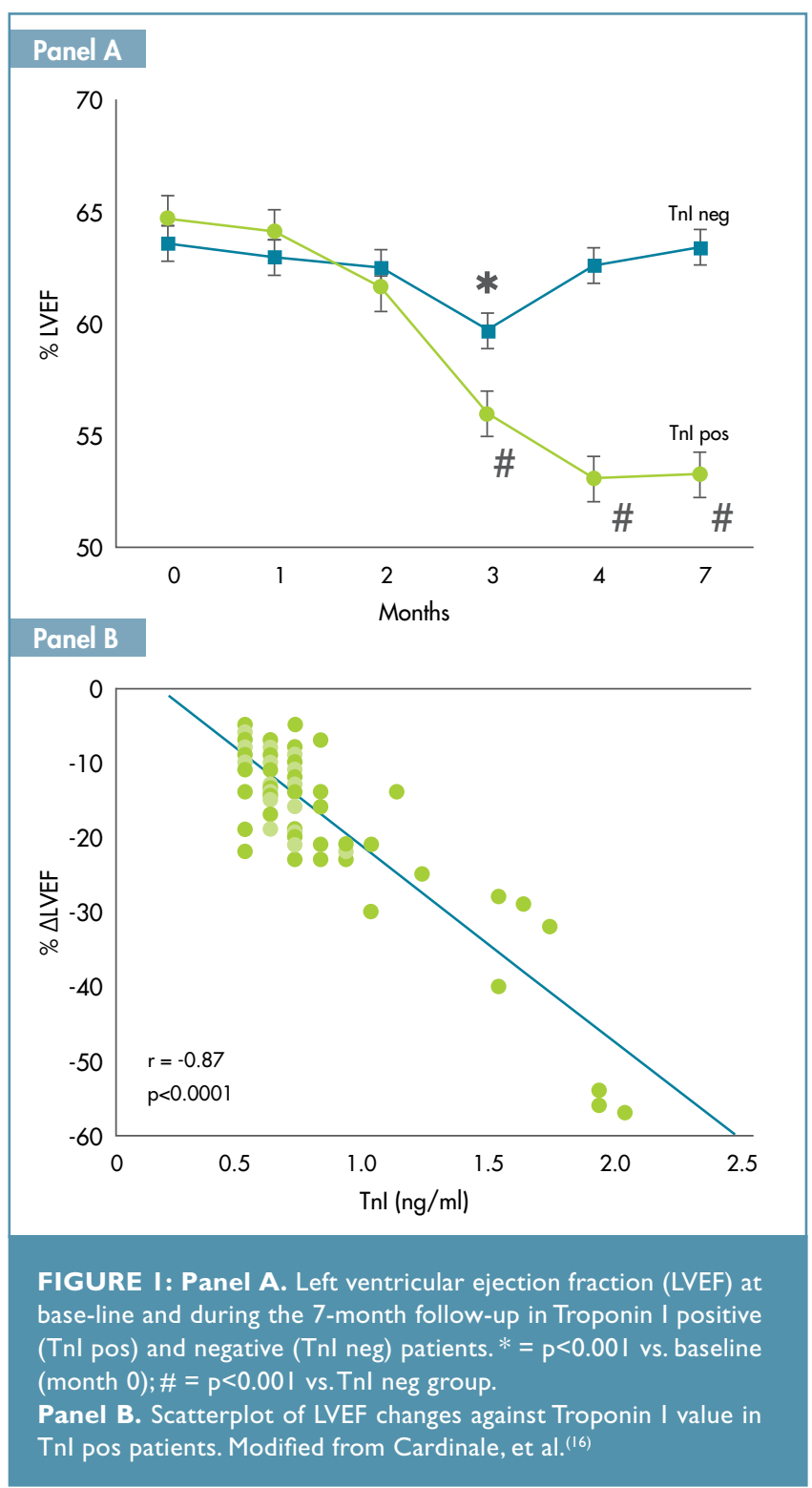


cancer patients, in whom Tnl was determined before CT, during the following three days after the end of CT (early evaluation) and after one month (late evaluation), three different troponin release patterns identified patients at different risk of cardiotoxicity. Troponin I was consistently negative in $70 \%$ of cases, positive at only early evaluation in $21 \%$, and positive at both early and late evaluations in $9 \%$ (Figure 2). ${ }^{(19)}$ During the 3.5 year follow-up, $1 \%$, $37 \%$, and $84 \%(p<0.001)$ of patients, respectively, experienced an adverse cardiac event. Thanks to its high negative predictive value (99\%), Tnl allows us to identify low-risk patients who will not require further cardiac monitoring. In contrast, $\mathrm{Tnl}$ positive patients require more stringent surveillance, particularly those showing a persistent $\mathrm{Tnl}$ increase. Indeed, these patients experienced a greater LVD and a higher incidence of cardiac events than patients showing only a transient increase in this marker. ${ }^{(19)}$

Troponin measurements have proven to be useful for the early detection of cardiotoxicity also in patients treated with a standard dose of CT. Auner, et al. ${ }^{(20)}$ reported a TnT increase in 15\% of patients treated with standard doses of AC. Patients with an elevated $\mathrm{Tn} T$ level showed a significantly greater absolute decrease in LVEF than those without an elevation in the marker (10\% vs. $2 \%$; $p=0.017$ ). A significant LVEF reduction was observed in Tnl positive patients treated with $\mathrm{AC}$ for leukemia. ${ }^{(21)}$ In addition, an increased TnT level, detected in the first 3-5 days following administration of standard doses of AC, predicted diastolic dysfunction in 34\% of patients. (22)

Troponins may also be utilised for the early detection of cardiac injury in patients undergoing treatment with new targeted anti-

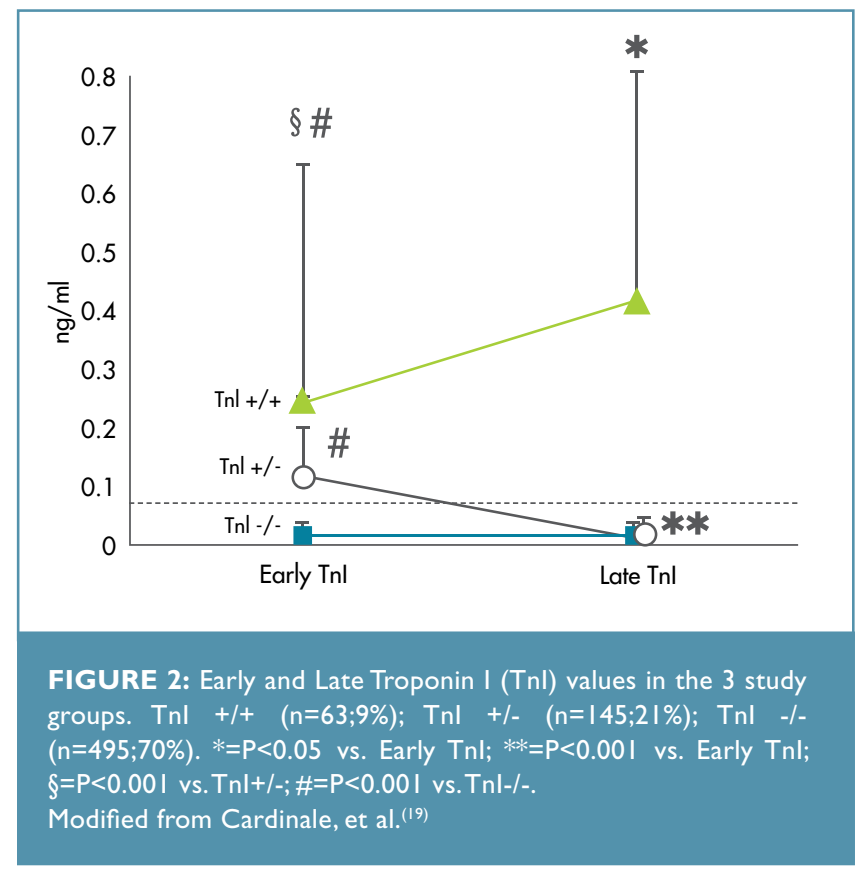

TABLE I: Major adverse cardiac events in the overall study population and in patients with elevated or normal Troponin I value. (Modified from Cardinale, et al. $\left.{ }^{(23)}\right)$

\begin{tabular}{|c|c|c|c|}
\hline & $\begin{array}{l}\text { Total } \\
(n=251)\end{array}$ & $\begin{array}{c}\text { Elevated } \\
\text { Troponin I } \\
(\mathrm{n}=36)\end{array}$ & $\begin{array}{c}\text { Normal } \\
\text { Troponin I } \\
(n=2 \mid 5)\end{array}$ \\
\hline Severe LVEF reduction ( $\leq 30 \%), \mathrm{n}(\%)$ & $7(3)$ & $6(17)$ & I (0.5) \\
\hline Cardiac death, $n$ (\%) & $0(0)$ & $0(0)$ & $0(0)$ \\
\hline Acute coronary syndrome, n (\%) & $2(1)$ & $2(5)$ & $0(0)$ \\
\hline Acute pulmonary oedema, n (\%) & I (0.5) & I (3) & $0(0)$ \\
\hline Heart failure, n (\%) & $7(3)$ & $7(19)$ & $0(0)$ \\
\hline Arrhythmias requiring treatment, $n$ (\%) & $5(2)$ & $2(8)$ & $3(1.4)$ \\
\hline Cumulative events & $22(9 \%)$ & $18(50 \%)$ & $4(2 \%) *$ \\
\hline
\end{tabular}

$L V E F=$ left ventricular ejection fraction. $* P<0.001$ vs. elevated Troponin (by Fisher exact test).

TABLE 2: Elevation of cardiac troponins in patients without overt ischaemic heart disease (Modified from Panteghini M. ${ }^{(26)}$ ).

\begin{tabular}{l} 
Acute rheumatic fever \\
Amyloidosis \\
Cardiac trauma (including contusion, ablation, pacing, firing, cardioversion, \\
catheterisation, cardiac surgery) \\
Cardiotoxicity from cancer therapy \\
Congestive heart failure \\
Critically ill patients \\
End-stage renal failure \\
Glycogen storage disease type II (Pompe's disease) \\
Heart transplantation \\
Haemoglobinopathy with transfusion haemosiderosis \\
Hypertension, including gestational \\
Hypotension, often with arhythmias \\
Hypothyroidism \\
Myocarditis/Pericarditis \\
Post-operative non-cardiac surgery \\
Pulmonary embolism \\
\hline Sepsis
\end{tabular}

cancer drugs. The role of Troponin I was assessed in 25 I breast cancer patients treated with trastuzumab.(23) $\mathrm{Tnl}$ was measured immediately before and immediately after each cycle, and resulted as having increased in 34 (I4\%) of patients. LVD occurred in 62\% of patients showing an increase of $\mathrm{Tnl}$ during trastuzumab treatment, and in only $5 \%$ of those with normal Tnl value $(p<0.00 \mathrm{l})$. Patients showing an increase of Tnl during trastuzumab treatment had a three-fold lower chance of recovery from cardiac dysfunction and had a higher incidence of cardiac events (Table I). In this study, therefore, Tnl was able to accurately identify patients at risk of developing LVD and, among them, those who will less likely recover 
from cardiotoxicity - despite optimised heart failure treatment thus possibly distinguishing between reversible and irreversible cardiac injury. In a recent study, the timing of maximum detectable $\mathrm{Tnl}$ value preceded maximum decline in LVEF in patients receiving both trastuzumab and lapatinib - a tyrosine-kinase inhibitor following AC-based CT.(24) In 86 patients with metastatic renal cancer treated with tyrosine-kinase inhibitor sunitinib or sorafenib and monitored prospectively with serial $\operatorname{TnT}$ measurements an increase in the markers was observed in 10\% of patients. Ninety percent of them showed a decrease in LVEF or regional contraction abnormalities. ${ }^{(25)}$ These data suggest that troponins may be useful for assessing cardiotoxicity in patients treated with both old and new antineoplastic treatments. Possibly, the release of troponin reflects a final common event for multiple cardiotoxic mechanisms. As many non-ischaemic pathophysiological conditions can cause myocardial necrosis, and therefore elevations in cardiac troponin concentrations (Table 2), other possible causes of troponin increase must obviously to be excluded. ${ }^{(26)}$

\section{Different timing of troponins increase}

Following acute ischaemic myocardial damage, troponins show a specific kinetic profile: range of times to initial elevation 3- 12 hours, lasting time up to 7 (Tnl) or 14 (TnT) days. ${ }^{(27)}$ On the contrary, the time course of troponin changes following $\mathrm{CT}$ is more variable and less clearly defined. Indeed, data from available studies show that troponins changes might be detected at various time intervals after CT administration, and are possibly due to different troponin release kinetics in response to different treatment schedules containing drugs with possible synergic toxic effect. $(14-16,19-23)$ Moreover, the sampling protocols used for measurement of troponins were not homogeneous in terms of cut-offs, timing of sampling and cardiac endpoints definition. Standardisation of routine troponin use in this clinical setting is a current need, and future research should focus on the best timing for sampling at which the maximal sensitivity and sensibility from a single sample of troponin can be obtained.

Moreover, additional factors may influence the pattern of troponin release; these include use of variable cumulative doses of anticancer drugs administered, method and timing of administration, and prior drugs exposure. ${ }^{(28,29)}$ In breast cancer patients treated with highdose AC-containing schedules, we found that the percentage of $\mathrm{Tnl}$ positivity progressively increased in parallel with the increasing number of the cycles performed, confirming that the risk of cardiotoxicity in AC-treated patients is dose dependent (Figure 3; panel A). ${ }^{(17)}$ Conversely, in breast cancer patients treated with trastuzumab, we found that the first increase in troponin was most frequent soon after the first cycle of trastuzumab and occurred only in patients previously treated with AC (Figure 3; panel B), suggesting a possible combined mechanism: Tnl rise in these patients possibly reflects $A C$ injury facilitated by trastuzumab, rather than a de-novo cardiac damage. ${ }^{(23)}$ This hypothesis might explain the different timing of the rise in $\mathrm{Tnl}$ in our studies. Many studies have also elucidated that troponin value can remain elevated for a period ranging from a few hours to several months, after the completion of CT. $(19,20,21,28,30)$ On the other hand, the exact time-point at which a negative troponin value reaches $100 \%$ of specificity for no further troponin release cannot yet be defined, ${ }^{(31)}$ therefore, repeated blood samples are required to pick out a possible troponin increase. This represents a possible limitation for using these markers in clinical practice. However, this strategy seems to be justified and absolutely cost-effective when negative values allow for the exclusion of most patients from a long-term monitoring programme with an expensive imaging technique.

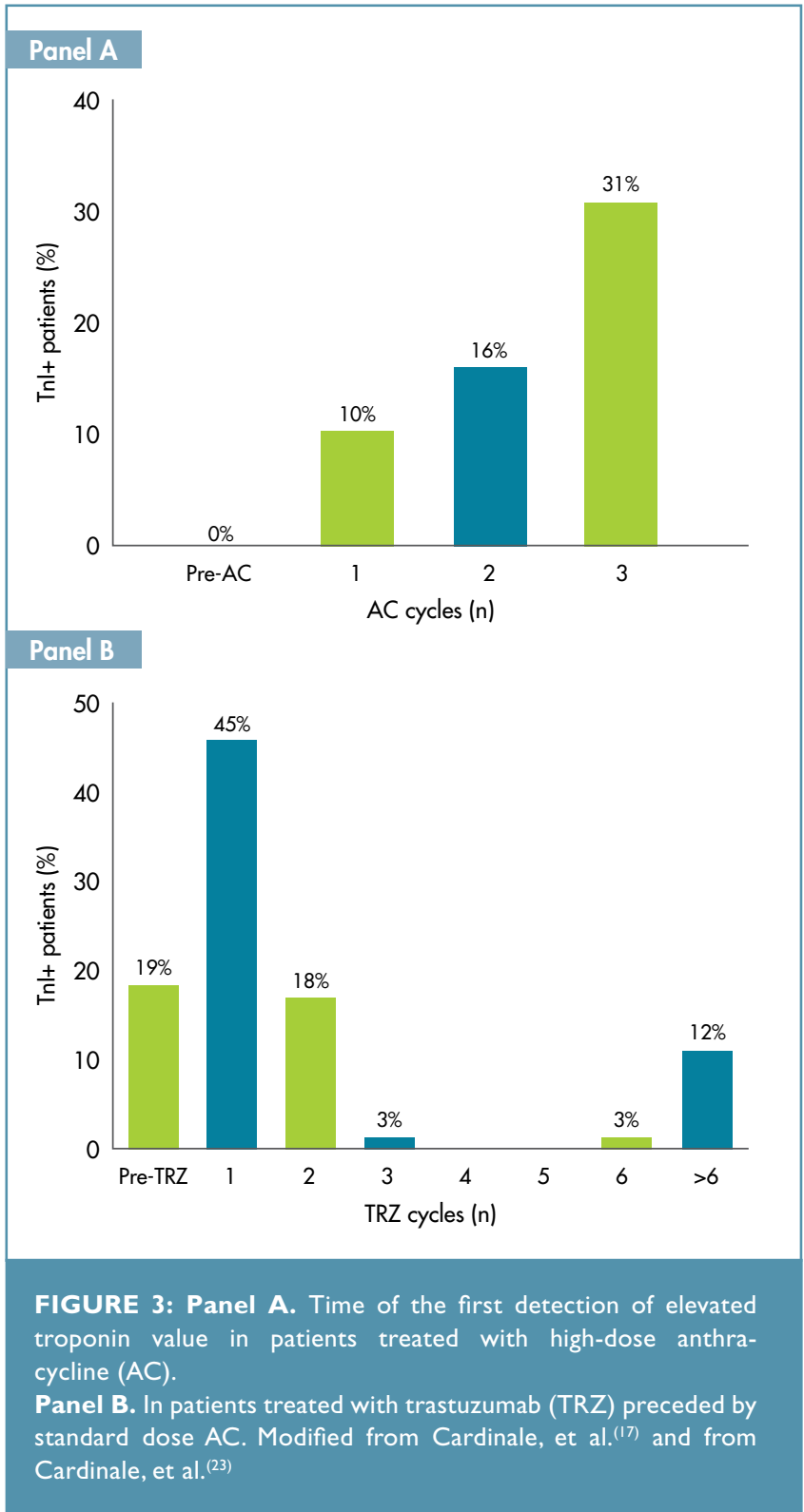




\section{High-sensitivity troponins}

Recently the technological evolution led to the generation of troponin assays with improved sensitivity, enabling a reliable detection of very low amounts of troponin. These assays are referred as "high sensitivity" troponins although definite criteria to define their analytical performance are still lacking. These tests could be of particular interest in the cardiotoxicity field, and may offer some advantages, as often we are dealing with a very low concentration of troponin, and it is of utmost importance to use systems showing high precision also at a very low level. ${ }^{(32)}$

The possible employment of high-sensitivity $(H S)$ troponins in this setting is provided by a recent multicentre study, in which the authors evaluated global and regional myocardial function by tissue Doppler and strain rate imaging, combined with HS Tnl, at baseline, and 3 and 6 months during $C T$ including $A C$ and trastuzumab. ${ }^{(33)}$ HS Tnl increased in $28 \%$ of patients. The decrease in left ventricular longitudinal strain, and a detectable HS Tnl, at 3 months were independent predictors of the development of cardiotoxicity at 6 months. On the other hand, changes in LVEF, diastolic function, and N-terminal pro-Brain Natriuretic Peptide (NT-proBNP), evaluated at the same time points, were not predictive of later LVD. Further larger investigations comparing HS Tn with traditional troponin assays are needed.

\section{Natriuretic peptides}

During the last 10 years several studies have assessed the clinical utility of natriuretic peptides (NP) in the diagnosis and risk assessment of patients with heart failure. Many studies have also investigated their possible role for the diagnosis and prediction of cardiotoxicity induced by anticancer drugs. The first observation, by Suzuky, et al., ${ }^{(34)}$ dates back to the end of the nineties: a persistent elevation of Brain Natriuretic Peptide (BNP) following AC administration was associated with the development of LVD, as compared to patients with only transient elevation. Afterwards, several papers were published, evaluating a total of more than I 200 patients, with different malignancy (haematologic and solid tumours), age (children and adults) and oncologic treatment (Table 3). ${ }^{(35-55)}$ In most of the studies an association between increased levels of NP and LVD was found. However, only few reports indicated NP as predictors of LVD after anticancer treatment. We conducted a retrospective study including 52 patients receiving high-dose chemotherapy for aggressive malignancies. ${ }^{(39)}$ Three distinct increase patterns were found, according to the NT-proBNP trend during the 6 blood samples taken in the 72 hours after CT (Figure 4; panel A). Patients who did not manifest significant echocardiographic changes recorded during followup were those who maintained low levels of NT-proBNP for all the time of sampling (Group A, 32\% of patients) or had only a transient increase (Group B, 36\% of patients). In patients in whom the level of NT-proBNP increased and remained elevated after 72 hours (Group C, 32\% of patients), we observed a deterioration of diastolic indexes (mitral deceleration time, isovolumetric relaxation time, and mitral E/A ratio) and a decrease in LVEF (from 62.8\% to 45.6; $\mathrm{P}<0.00 \mathrm{I}$ ) (Figure 4; panel B), in the following months after the completion of $\mathrm{CT}$. A strong relationship between NT-proBNP value at 72 hours and LVEF changes at 12 months versus baseline $(r=0.78 ; p<0.00 I)$ was found. These results are consistent with more recent reports in which a NT-proBNP increase during ACcontaining regimens predicted both significant LVD decline and overt HF in adults and paediatric populations. ${ }^{(51-55)}$ However, the heterogeneousness of data (different populations, sample sizes, kind of NP used, method of measurement, cut-offs, cardiac endpoints, duration of follow-up) makes the comparison of the results from the different studies quite difficult, and it does not allow us to draw definite conclusions or indications regarding the appropriate use of these biomarkers in clinical practice.

\section{Other proposed biomarkers}

Other molecules have been proposed as biomarkers for cardiovascular injury related to $\mathrm{CT}$-induced toxic effect.

New markers of myocardial ischaemia and necrosis such as fatty acid binding protein and glycogen phosphorylase isoenzyme BB (brain) have been reported to also increase after CT with $A C$. $^{(56,57)}$ However, although these markers are highly sensitive, they have relatively low cardiac specificity and available data are insufficient to propose their use in the follow-up of oncology patients.

The increase of markers of inflammation, such as cytokines, has also been described after $\mathrm{CT}$. Mercuro, et al. ${ }^{(58)}$ reported a correlation between interleukine-6 (IL-6) increase and early changes in systolic function when analysed by tissue Doppler imaging in a small population of patients treated with CT containing epirubicin. More recently the same group showed that the angiotensin II receptor blocker telmisartan can reduce AC-induced radical species, antagonise the inflammation, and reverse the early myocardial impairment. ${ }^{(59)}$ These preliminary results warrant confirmation by further, larger prospective investigations.

Because chemotherapeutic agents can induce endothelial activation, several studies have analysed a possible role of molecules deriving from endothelial damage as markers of cardiovascular toxicity. Nuver, et al. ${ }^{\left({ }^{60}\right)}$ reported an increased level of endothelial dysfunction markers (tissue-type plasminogen activator and plasminogen activator inhibitor type I) in patients treated with cisplatin- 


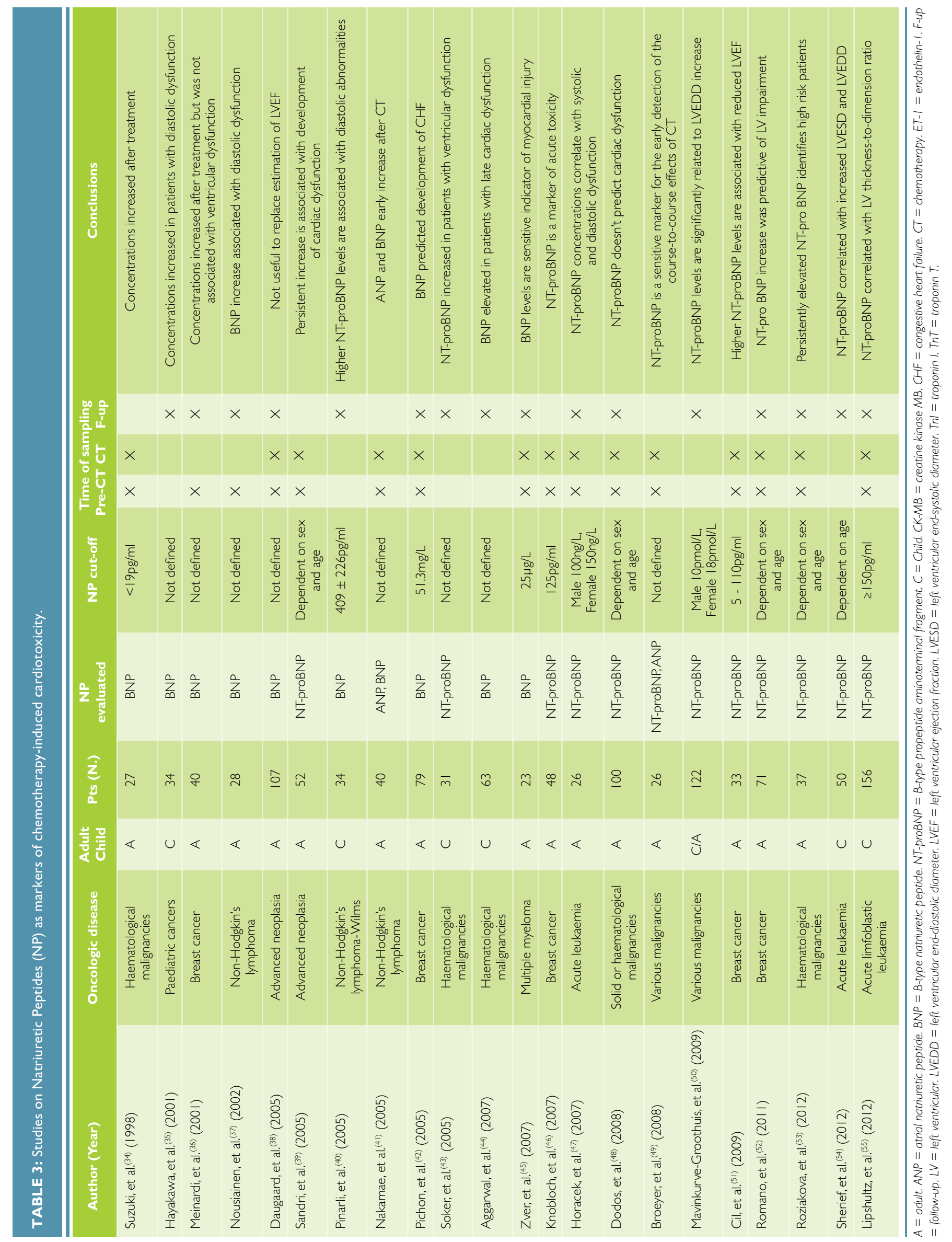




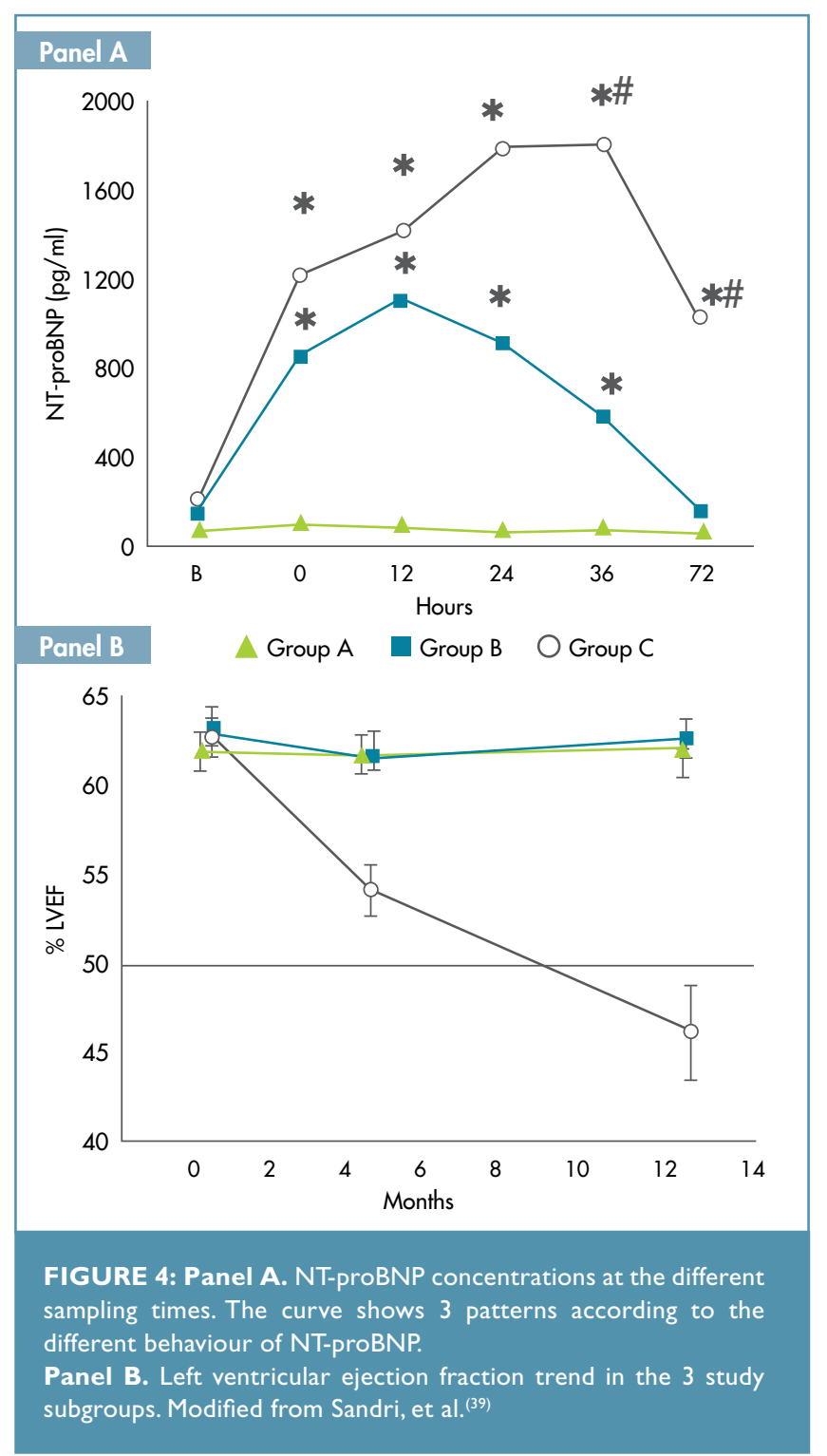

based CT for testicular cancer many years before. In a similar population Vaughn, et al.(61) reported that long-term cancer survivors treated with CT showed increased markers of endothelial injury compared to CT-naive survivors. These findings suggest that CT may induce endothelial dysfunction and accelerate atherosclerotic processes, leading to an increased risk for future cardiovascular diseases. ${ }^{(62)}$ However, at present, no correlation with long-term cardiovascular events has been demonstrated, and the predictive role of these markers has yet to be defined.

\section{PREVENTION OF CARDIOTOXICITY}

\section{Current possible strategies}

To prevent cardiotoxicity, several preventive measures are currently used, including limitation of cumulative $A C$ dose, slowing down of
AC administration, and use of less cardiotoxic AC analogues. However, the addition of cardioprotectants or nutritional supplements and detection of early signs of cardiotoxicity by biomarkers are the two most promising strategies. ${ }^{(11,63,64)}$

\section{Addition of cardioprotectants to AC treatment}

Carvedilol, a beta-blocker with alfa-I-blocking vasodilatory properties, has also showed strong antioxidant activity that lends it a cardioprotective effect against doxorubicin.(63) This favourable effect was confirmed in an in-vitro study ${ }^{(65)}$ and in a randomised study in which prophylactic use of carvedilol prevented LVD and reduced mortality in a small population of patients treated with AC. (66)

Dexrazoxane, an iron-chelating agent, significantly reduced ACrelated cardiotoxicity in adults with different solid tumours and in children with acute lymphoblastic leukaemia and Ewing's sarcoma. ${ }^{(15,67,68)}$ Moreover, the same agent was more effective than an AC prolonged infusion in preventing cardiotoxicity in 23 Ewing's sarcoma patients treated with doxorubicin. ${ }^{(69)}$ Nevertheless, dexrazoxane is not routinely used in clinical practice and it is recommended as a cardioprotectant by the American Society of Clinical Oncology only in patients with metastatic breast cancer who have already received more than $300 \mathrm{mg} / \mathrm{m}^{2}$ of doxorubicin. This might be explained by the suspicion of interference with antitumor efficacy of $A C$ and facilitation of the occurrence of secondary malignancies as well as by its possible myelosuppressor effect. However, meta-analyses didn't find a significant difference in terms of antitumor efficacy and of occurrence of secondary malignancies between patients who were treated with and without dexrazoxane. ${ }^{(63,67,70,71)}$

Many other chemical agents have been evaluated such as coenzyme Q I O, carnitine, N-acetylcysteine, the antioxidant vitamins E and C, erythropoietin, the endothelin-I receptor antagonist bosentan, the lipid-lowering agents probucol and statins and some of them showed promising results. Other iron-chelating agents, such as desferoxamine and EDTA, are also of interest (Table 4). Although preliminary findings show that all these agents may have cardioprotective effects their utility in preventing CMP need to be confirmed by further investigation. $(3,11,63,67)$

The role of biomarkers in the prevention of cardiotoxicity A pharmacologic preventive approach extended to all cancer patients treated with $C T$ has a high cost-benefit ratio and exposes patients to possible side effects, including a potential antagonistic effect to antitumor activity of the treatment also in patients less 
prone to develop cardiotoxicity. A preventive therapy in selected high-risk patients only, identified by an increase in cardiac biomarkers during and/or after CT, may represent a reasonable alternative. The possibility of identifying patients at high-risk of developing cardiotoxicity by cardiac biomarkers provides a rationale for the development of tailored preventive strategies directed at reducing the clinical impact of cardiotoxicity. Two different strategies could be carried out:

- Use of specific cardiologic treatments given to cancer patients during the oncologic treatment in the attempt to prevent or blunt the rise of these markers or interfere with their persistence after first increase;

- Use of cardiologic treatments given only to selected cancer patients identified by an increase in these markers during $\mathrm{CT}$.

A recent prospective study reported that valsartan, an angiotensin II receptor blocker, given at the same time as doxorubicin was able to prevent an increase in atrial natriuretic peptide, BNP, and left ventricular diastolic diameter and prolongation and dispersion in QTc interval in patients with non-Hodgkin's lymphoma. ${ }^{(72)}$ Lipshultz, et al. ${ }^{(68)}$ reported that $\mathrm{TnT}$ elevation occurred significantly more frequently in leukaemic children receiving doxorubicin alone than in those in whose doxorubicin was administered in association with dexrazoxane ( $50 \%$ vs. $21 \%$, respectively; $p<0.00$ I).
The usefulness of $\mathrm{Tnl}$ screening for a selection of patients requiring prophylactic cardioprotective therapy was investigated in a randomised, controlled trial carried out at our institute. ${ }^{(30)}$ The cardioprotective effects of enalapril were evaluated in 413 patients treated with high-dose AC. The II 4 (24\%) patients showing early Tnl increase were randomised to enalapril or no treatment. Enalapril was initiated I month after the completion of CT, titrated at the maximal tolerated dose, and continued for I year. In the enalapril-treated group, LVEF did not change during the follow-up period. Conversely, in patients not receiving enalapril a progressive reduction in LVEF and an increase in end-diastolic and endsystolic volumes were observed (Table 5). Moreover, a significantly lower incidence of adverse cardiac events was found in enalapriltreated patients than in controls at a I-year follow-up (2\% vs.52\%; $\mathrm{p}<0.00 \mathrm{I})$. ${ }^{(30)}$

Going from clinical research to the real world we applied this strategy to our daily clinical practice. A treatment with enalapril is usually started in all patients showing a Tnl increase after treatment with both old and new anticancer drugs. In more than I 350 treated patients no case of significant reduction in LVEF (below 50\%) was observed during a mean follow-up of 4.6 years (unpublished data). These results confirm that this targeted prophylactic approach can be really effective in preventing cardiotoxicity and may be easily and safely applied in daily clinical practice.

TABLE 4: Therapies for reducing anthracycline toxicity.

\begin{tabular}{|c|c|c|c|}
\hline Agent & Class & Mechanism & \\
\hline Carvedilol & b-Adrenergic antagonist & Prevention of free radical formation; prevention of depletion of endogenous antioxidants & Humans \\
\hline Valsartan & Angiotensin II receptor blocker & Inhibition of Angiotensin II effects & Humans \\
\hline Dexrazoxane & Chelating agent & Prevention of free radical formation; binding to iron inhibits DNA topoisomerase & Humans \\
\hline Coenzyme Q10 & Dietary supplement & Antioxidant & Humans \\
\hline Carnitine & Dietary supplement & Antioxidant; transfer of long chain fatty acids into mitochondria & Humans \\
\hline N-acetylcysteine & Mucolytic agent & Promotion of endogenous antioxidant synthesis & Humans \\
\hline Vitamin A, C and E & Nutrient & Antioxidant & Animal model/Humans \\
\hline Erythropoietin & Hormone & Apoptosis prevention & Animal model \\
\hline Bosentan & Endothelin-I receptor antagonist & $\begin{array}{l}\text { Decrease inflammatory markers (TNF- } \alpha \text { ) and the expression of apoptotic } \\
\text { signaling proteins }\end{array}$ & Animal model \\
\hline Probucol & Lipid-lowering agent & Promotion of endogenous antioxidant synthesis & Animal model \\
\hline Fluvastatin & Statin & Antioxidant & Animal model \\
\hline Glutathione & Tripeptide thiol & Antioxidant & Animal model \\
\hline Selenium & Trace element & Antioxidant; anticarcinogenic action & Animal model \\
\hline Amifostine & Cytoprotective agent & Antioxidant; scavenges reactive oxygen species & Animal model \\
\hline Desferoxamine & Iron-chelating agent & Production of reactive oxygen species & Animal model \\
\hline
\end{tabular}


TABLE 5: Echocardiographic parameters during the study period (Modified from Cardinale, et al. ${ }^{(30)}$ )

\begin{tabular}{|c|c|c|c|c|c|c|c|}
\hline & & Baseline & Rand. & 3 months & 6 months & 12 months & P value \\
\hline \multirow[t]{2}{*}{ EDV (ml) } & ACEl-group & $101.7 \pm 27.4$ & $100.2 \pm 26.1$ & $98.1 \pm 27.8$ & $97.5 \pm 24.5$ & $101.1 \pm 26.4$ & \multirow{2}{*}{0.045} \\
\hline & Controls & $103.2 \pm 20.1$ & $103.9 \pm 21.0$ & $106.4 \pm 21.0$ & $107.1 \pm 23.9$ & $104.2 \pm 25.6$ & \\
\hline \multirow[t]{2}{*}{ ESV (ml) } & ACEl-group & $38.6 \pm 10.8$ & $38.7 \pm 10.4$ & $37.3 \pm 10.9$ & $37.4 \pm 10.3$ & $38.5 \pm 11.2$ & \multirow{2}{*}{$<0.001$} \\
\hline & Controls & $38.8 \pm 10.2$ & $40.5 \pm 12.2$ & $49.8 \pm 17.6$ & $51.8 \pm 16.9$ & $54.4 \pm 20.1^{\#}$ & \\
\hline \multirow[t]{2}{*}{ LVEF (\%) } & ACEl-group & $61.9 \pm 2.9$ & $61.1 \pm 3.2$ & $61.9 \pm 3.3$ & $61.6 \pm 3.9$ & $62.4 \pm 3.5$ & \multirow{2}{*}{$<0.001$} \\
\hline & Controls & $62.8 \pm 3.4$ & $61.8 \pm 4.3$ & $54.2 \pm 8.1$ & $51.9 \pm 7.9$ & $48.3 \pm 9.3^{\#}$ & \\
\hline
\end{tabular}

${ }^{*} P$ value for repeated measures analysis of variance. ${ }^{*} p<0.001$ vs. baseline. EDV $=$ end-diastolic volume. ESV = end-systolic volume. LVEF $=$ left ventricular ejection fraction Rand. $=$ randomisation.

The role of biomarkers in clinical trials

Measurement of cardiac biomarkers, and in particular of troponins, could allow for an early and objective diagnosis of cardiac toxicity in clinical trials evaluating cardiac safety of new antitumoral agents. The usefulness of $\mathrm{Tnl}$ monitoring in patients treated with developing molecular targeted therapies has recently been highlighted in phase I trials, by Ederhy, et al. ${ }^{(73)}$ Ninety patients with solid metastatic tumours were treated with new anti-VEGF monoclonal inhibitors and tyrosine kinase inhibitors in a phase I trial. All patients were also monitored with serial Tnl measurement. Ten of them ( I I\%) showed an increase in $\mathrm{Tnl}$ during the cancer treatment. These underwent echocardiography, cardiac MR, CT scan, and coronary angiography to exclude other possible causes of Tnl rise. Normalisation of $\mathrm{Tnl}$ values was obtained with a treatment associating beta-blockers (BB) and aspirin; then all patients were re-challenged with the study drug. No patient experienced any new increase of Tnl, and no cardiac events occurred during the subsequent observation period (mean follow-up: 3 months). The authors concluded that the use of Tnl allows for the early identification of patients more prone to develop cardiotoxicity when exposed to a new drug in cancer clinical trials. These data suggest that patients showing myocardial injury, revealed by the increase in Tnl, should be treated with a prophylactic therapy in order to prevent overt cardiotoxicity and should not be excluded from continuing oncologic treatment. Accordingly, the definition of cardiotoxicity in cancer clinical trials, still based on the evidence of symptomatic heart failure or asymptomatic decrease in LVEF, should be changed moving from a late clinical to an early subclinical definition. As a result the apparent increase in the incidence of subclinical cardiotoxicity due to the low threshold of detection of cardiac injury will be counterbalanced by a lower incidence of clinical cardiotoxicity with a raise in net clinical benefit of the new antitumoral treatment.

\section{TREATMENT OF CARDIOTOXICITY}

Anthracyclines-induced cardiotoxicity

Historically, AC-induced cardiomyopathy (CMP) was believed to be refractory to conventional therapy. This opinion was however based either on findings reported in old studies, in which standard therapy included only the use of digoxin and diuretics, or in studies with very small sample sizes. ${ }^{(64)}$ The response to modern HF therapy of patients with AC-induced CMP has never been fully evaluated because these patients have typically been excluded from large randomised trials investigating novel HF therapies. As a result, the use of angiotensin-converting enzyme inhibitors (ACEI) and $\mathrm{BB}$ in this particular clinical setting remain a matter of debate. Moreover the different etiology and age distribution of this kind of CMP when compared with the more frequent ischaemic or idiopathic CMPs raise some concern whether the use of ACEl and $\mathrm{BB}$ - recommended for other forms of CMPs - can be directly transferred to this particular clinical setting with similar long-term benefits. Finally as LVD due to anticancer drugs is in most cases asymptomatic many authors have suggested that screening programmes should look for overt HF and current management of LVD induced by anticancer therapy mainly focuses on treatment of symptomatic patients. ${ }^{(74)}$ This is probably due to the fact that there are special concerns in using ACEI and BB in such a population probably for the fear of unknown pharmacologic interactions. Because cancer patients are considered frail the tendency is not to treat them aggressively. Hence, evidence-based recommendations for the management of cancer patients who develop asymptomatic or symptomatic LVD after CT are still lacking.

In a recent study, the response to modern HF therapy, including ACEI and BB has been prospectively evaluated in 201 patients with AC-induced CMP. ${ }^{(6)}$ In this study the time elapsed from the end of CT to the start of HF therapy (time-to-treatment) including ACEI 
and, when tolerated, BB was a critical variable for recovery from LVD. Indeed, a strong inverse relationship between time-totreatment and LVEF improvement in response to HF therapy was observed $(R=-0.66 ; p<0.00 I)$. In particular, the likelihood of obtaining a complete LVEF recovery was higher in patients in whom HF treatment was initiated because of LVD detection within 2 months after the end of CT. After this time limit the percentage of complete LVD recovery progressively decreased and only partial recovery was observed after 6 months. When LVD was detected after 12 months the possibility of obtaining at least a partial recovery was completely exhausted (Figure 5). Notably patients showing complete LVD recovery had a significantly lower rate of adverse cardiac events than those who did not recover (5\% vs. 30\%). Moreover complete recovery was more frequently observed in patients with asymptomatic LVD than in those with symptoms of HF (56\% vs. II\%; $p<0.001)$. ${ }^{(6)}$ The monitoring of cardiotoxicity based exclusively on symptoms evaluation may therefore hinder the opportunity to detect LVD early in a still asymptomatic and reversible stage. It is also likely that in most previously published studies the poor response to HF therapy was due to undertreatment with $\mathrm{ACE}$ and $\mathrm{BB}$ and to late beginning of HF therapy (in most cases it was started more than 12 months after the end of chemotherapy) when LVD has been shown to be no longer reversible. This underlines that an early detection of cardiotoxicity is of pivotal importance and that a prompt therapy based on the association of $\mathrm{ACEl}$ and $\mathrm{BB}$ should always be considered and undertaken in all cases of anthracycline-induced CMP.

\section{Trastuzumab-induced cardiotoxicity}

The incorporation of trastuzumab, a monoclonal antibody targeting human epidermal growth factor receptor 2, to the standard regimens for treatment of breast cancer that over-expresses HER2 receptors - a variant of the disease generally associated with a poor prognosis - has led to a major improvement in outcome. ${ }^{(75)}$ Its use however has resulted in an unexpectedly high rate of cardiotoxicity which occurs in up to $7 \%$ of patients when used as monotherapy and in $28 \%$ of patients when associated with $A C$. $^{(1)}$

Trastuzumab causes a different form of cardiotoxicity from that typical of AC: It is not dose-dependent; AC-typical ultra structural changes are not seen on cardiac biopsy specimens; and it is not caused by oxidative mechanisms. ${ }^{(75)}$ The clinical outcome of patients who develop trastuzumab-induced cardiotoxicity seems more favourable and cardiac function generally improves after withdrawal on removal of the agent and initiation of HF therapy. Treatment of trastuzumab-related cardiotoxicity is a still controversial issue. Most patients (60-80\%) significantly improve their LVD when treated with drugs commonly used for HF such as ACEl and BB. (76) As patients who developed LVD in the adjuvant trastuzumab trials were not treated in a systematic manner the natural history of trastuzumab cardiotoxicity is currently unknown and evidencebased recommendations for its management are still lacking. Whether trastuzumab-related LVD has a course that is modified with $\mathrm{ACEl}$ and $\mathrm{BB}$ is still uncertain. ${ }^{(77)}$

Follow-up data from large trials show that in many patients treated with anthracycline and sequentially with trastuzumab LVD does

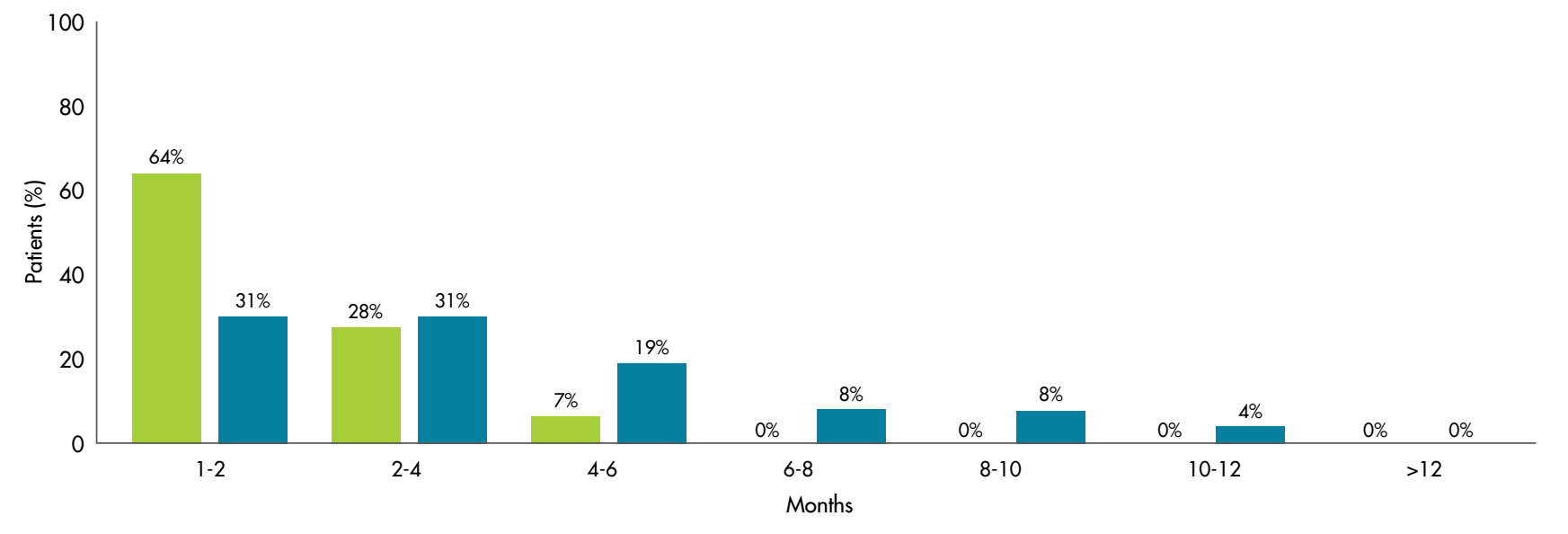

FIGURE 5: Percentage of patients showing a complete (green bars) or partial (blue bars) left ventricular ejection fraction recovery according to the time elapsed from anthracyclines administration to start of heart failure therapy. Modified from Cardinale, et al.(6) 
not recover; that up to two-thirds of patients continue to receive cardiac medications after complete functional recovery; and that many patients show a LVEF lower than baseline despite optimal HF therapy. ${ }^{(78)}$ For these reasons some uncertainties on resuming trastuzumab after cardiac function recovery still exist. This is particularly true for patients treated with curative intent while the risk of developing cardiac impairment in the metastatic setting is largely counterbalanced by the benefit of trastuzumab.

\section{CONCLUSION}

At present, anticancer treatment-induced cardiotoxicity still remains a serious problem strongly impacting both quality of life and over- all survival of cancer patients. The most effective approach to minimise cardiotoxicity lays in early detection and prompts prophylactic treatment initiation. The role of $\mathrm{Tnl}$ in identifying patients at high-risk of cardiotoxicity and their treatment with ACEl is clearly emerging as an effective strategy against this complication (Figure 6). In patients developing LVD after anticancer therapy a complete LVEF recovery and a reduction of cardiac events may be obtained only when LVD is detected early and a treatment with $\mathrm{ACEl}$, possibly in combination with $\mathrm{BB}$, is promptly initiated. ${ }^{(79)}$

\section{Conflict of interest: none declared.}

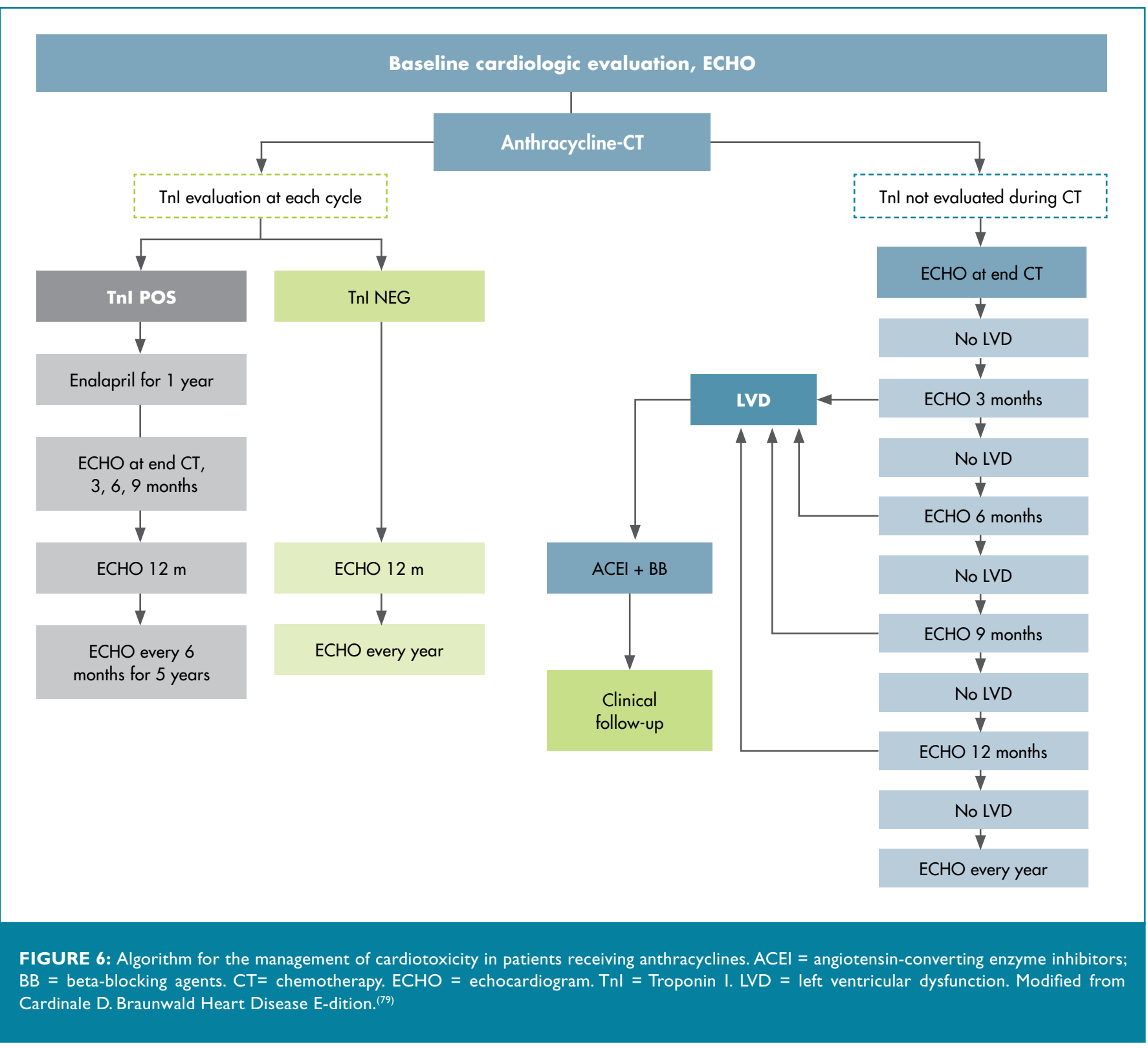


I. Yeh ETH, Bickford C. Cardiovascular complications of cancer therapy. J Am Coll Cardiol 2009;53:2231-47.

2. Jones RL, Ewer MS. Cardiac and cardiovascular toxicity of non-antracyclines anticancer drugs. Expert Rev Anticancer Ther 2006;6:1229-49.

3. Barry E, Alvarez JA, Scully RE, et al. Anthracycline-induced cardiotoxicity: course, pathophysiology, prevention and management. Expert Opin Pharmacother 2007:8: 1039-58

4. Hunt SA, Abraham WT, Chin MH, et al. Focused update incorporated into the ACC/AHA 2005 guidelines for the diagnosis and management of heart failure in adults. A report of the American College of Cardiology Foundation/American Heart Association Task Force on Practice Guidelines Developed in collaboration with the International Society for Heart and Lung Transplantation. J Am Coll Cardiol 2009;53:el-e90.

5. Altena R, Perik PJ, van Veldhuisen DJ, et al. Cardiovascular toxicity caused by cancer treatment: strategies for early detection. Lancet Oncol 2009; 1 0:39 I-9.

6. Cardinale D, Colombo A, Lamantia G, et al. Anthracycline-induced cardiomyopathy. Clinical relevance and response to pharmacologic therapy. J Am Coll Cardiol 2010;55:213-20.

7. Healey Bird BRJ, Swain SM. Cardiac toxicity in breast cancer survivors: review of potential cardiac problems. Clin Cancer Res 2008; 14:14-24.

8. Nielsen D, Jensen JB, Dombernowsky P, et al. Epirubicin cardiotoxicity: a study of I 35 patients with advanced breast cancer. J Clin Oncol 1990;8:1806-10.

9. Jensen BV, Skovsgaard T, Nielsen SL. Functional monitoring of anthracycline cardiotoxicity: a prospective, blinded, long-term observational study of outcome in 120 patients. Ann Oncol 2002; 13:699-709.

10. Plana JC. Chemotherapy and the heart. Rev Esp Cardiol 201 I:64:409-I 5.

11. Geisberg CA, Sawyer DB. Mechanisms of anthracycline cardiotoxicity and strategies to decrease cardiac damage. Curr Hypertens Rep 2010; 1 2:404- 10.

12. O'Brien PJ. Cardiac troponin is the most effective translational safety biomarker for myocardial injury in cardiotoxicity. Toxicology 2008;245:206- 18

13. Urbanova D, Urban L, Carter A, et al. Cardiac troponins - biochemical markers of cardiac toxicity after cytostatic therapy. Neoplasma 2006;53: 183-90.

14. Lipshultz SE, Rifai N, Sallan SE, et al. Predictive value of cardiac Troponin T in paediatric patients at risk for myocardial injury. Circulation 1997;96:264I-8.

15. Lipshultz SE, Scully RE, Lipsitz SR, et al. Assessment of dexrazoxane as a cardioprotectant in doxorubicin-treated children with high-risk acute lymphoblastic leukaemia: long-term follow-up of a prospective, randomised, multicentre trial. Lancet Oncol 2010; 1 1:950-61.

16. Cardinale D, Sandri MT, Martinoni A, et al. Left ventricular dysfunction predicted by early troponin I release after high-dose chemotherapy. J Am Coll Cardiol 2000;36:517-22

17. Cardinale D, Sandri MT, Martinoni A, et al. Myocardial injury revealed by plasma Troponin I in breast cancer treatment with high dose chemotherapy. Ann Oncol 2002;13;710-5.

18. Sandri MT, Cardinale D, Zorzino L, et al. Minor increases in plasma Troponin I predict decreased left ventricular ejection fraction after high-dose chemotherapy. Clin Chem 2003;49:248-52.

19. Cardinale D, Sandri MT, Colombo A, et al. Prognostic value of Troponin I in cardiac risk stratification of cancer patients undergoing high-dose chemotherapy. Circulation 2004; 109:2749-54.

20. Auner HW, Tinchon C, Linkesch W, et al. Prolonged monitoring of Troponin T for the detection of anthracycline cardiotoxicity in adults with haematological malignancies. Ann Haematol 2003;82:21 8-22.

21. Specchia G, Buquicchio C, Pansini N, et al. Monitoring of cardiac function on the basis of serum troponin I levels in patients with acute leukaemia treated with anthracyclines. J Lab Clin Med 2005; I 45:21 2-20.

22. Kilickap S, Barista I, Kabul E, et al. cTnT can be a useful marker for early detection of anthracycline cardiotoxicity. Ann Oncol 2005; | 6:798-804
23. Cardinale D, Colombo A, Torrisi R, et al. Trastuzumab-induced cardiotoxicity: clinical and prognostic implications of Troponin I evaluation. I Clin Oncol 2010;28:3910-6

24. Morris PG, Chen C, Steingart RM, et al. Troponin I and C-reactive protein are commonly detected in patients with breast cancer treated with dose-dense chemotherapy incorporating trastuzumab and lapatinib. Clin Cancer Res 2011;17:3490-9.

25. Schmidinger M, Zielinski CC, VogI UM, et al. Cardiac toxicity of sunitinib and sorafenib in patients with metastatic renal cell carcinoma. J Clin Oncol 2008;26:5204-12.

26. Panteghini $M$, Panteghini $M$. The new definition of myocardial infarction and the impact of troponin determination on clinical practice. Int J Cardiol 2006; 106 : 298-306

27. Adams JE, Abendschein DR, Jaffe AS. Biochemical markers of myocardial injury. Is MB creatinine kinase the choice for the 1990? Circulation 1993:88:750-63.

28. Adamcova M, Simunek T, Kaiserova, et al. In vitro and in vivo examination of cardiac troponins as biochemical markers of drug-induced cardiotoxicity. Toxicology 2007;237:218-28.

29. Germanakis I, Anagnostatou N, Kalmanti M. Troponins and natriuretic peptides in the monitoring of anthracycline cardiotoxicity. Paediatric Blood Cancer 2008:51:327-33.

30. Cardinale D, Colombo A, Sandri MT, et al. Prevention of high-dose chemotherapy-induced cardiotoxicity in high-risk patients by angiotensin-converting enzyme inhibition. Circulation 2006; | |4:2474-8|

31. Dolci A, Dominici R, Cardinale D, et al. Biochemical markers for prediction of chemotherapy-induced cardiotoxicity: systematic review of the literature and recommendations for use. Am J Clin Pathol 2008; 1 30;688-95.

32. Christenson RH, Phillips D. Sensitive and high sensitivity next generation cardiac troponin assays: more than just a name. Pathology 201 1;43:213-9.

33. Sawaya $\mathrm{H}$, Plana JC, Januzzi JL, et al. Early detection and predictor of cardiotoxicity in chemotherapy-treated patients. Am J Cardiol 201 1;107:1375-80.

34. Suzuki T, Hayashi D, Yamazaki T, et al. Elevated B-type natriuretic peptide levels after anthracycline administration. Am Heart J 1998;136:362-3.

35. Hayakawa $\mathrm{H}$, Komada $\mathrm{Y}$, Hirayama M, et al. Plasma levels of natriuretic peptides in relation to doxorubicin-induced cardiotoxicity and cardiac function in children with cancer. Med Paediatric Oncol 2001;37:4-9.

36. Meinardi MT, Van Veldhuisen DJ, Gietema JA, et al. Prospective evaluation of early cardiac damage induced by epirubicin-containing adjuvant chemotherapy and loco regional radiotherapy in breast cancer patients. J Clin Oncol $2001 ; 19: 2746-53$

37. Nousiainen T, Vanninen E, Jantunen E, et al. Natriuretic peptides during the development of doxorubicin-induced left ventricular diastolic dysfunction. J Intern Med 2002;251:228-34

38. Daugaard G, Lassen $U$, Bie $P$, et al. Natriuretic peptides in the monitoring of anthracycline induced reduction in left ventricular ejection fraction. Eur J Heart Fail 2005;7:87-93

39. Sandri MT, Salvatici M, Cardinale D, et al. N-terminal pro-B-type natriuretic peptide after high-dose chemotherapy: a marker predictive of cardiac dysfunction? Clin Chem 2005;51:1 405-10.

40. Pinarli FG, Oğuz A, Tunaoğlu FS. Late cardiac evaluation of children with solid tumours after anthracycline chemotherapy. Paediatric Blood Cancer 2005;44: 370-7.

4I. Nakamae H, Tsumura K, Terada Y, et al. Notable effects of angiotensin II receptor blocker, valsartan, on acute cardiotoxic changes after standard chemotherapy with cyclophosphamide, doxorubicin, vincristine and prednisolone. Cancer 2005; 104:2492-8.

42. Pichon MF, Cvitkovic F, Hacene K, et al. Drug-induced cardiotoxicity studied by longitudinal B-type natriuretic peptide assays and radionuclide ventriculography. In Vivo 2005; 19:567-76. 


\section{REFERENCES}

43. Soker M, Kervancioglu M. Plasma concentrations of NT-pro-BNP and cardiac Troponin I in relation to doxorubicin-induced cardiomyopathy and cardiac function in childhood malignancy. Saudi Med J 2005;26:1 197-202.

44. Aggarwal S, Pettersen MD, Bhambhani K, et al. B-type natriuretic peptide as a marker for cardiac dysfunction in anthracycline-treated children. Paediatric Blood Cancer 2007;49:812-6.

45. Zver S, Zadnik V, Bunc M, et al. Cardiac toxicity of high-dose cyclophosphamide and melphalan in patients with multiple myeloma treated with tandem autologous haematopoietic stem cell transplantation. Int J Haematol 2008;88:227-36.

46. Knobloch K, Tepe J, Lichtinghagen R, et al. Simultaneous haemodynamic and serological cardiotoxicity monitoring during immunotherapy with trastuzumab. Int J Cardiol 2008; 125: | 13-5.

47. Horacek JM, Pudil R, Jebavy $L$, et al. Assessment of anthracycline-induced cardiotoxicity with biochemical markers. Exp Oncol 2007;29:309-13.

48. Dodos F, Halbsguth T, Erdmann E, et al. Usefulness of myocardial performance index and biochemical markers for early detection of anthracycline-induced cardiotoxicity in adults. Clin Res Cardiol 2008;97:3। 8-26.

49. Broeyer FJ, Osanto S, Ritsema van Eck HJ, et al. Evaluation of biomarkers for cardiotoxicity of anthracyclin-based chemotherapy. J Cancer Res Clin Oncol 2008; 134:961-8.

50. Mavinkurve-Groothuis AM, Groot-Loonen J, Bellersen L, et al. Abnormal NT-proBNP levels in asymptomatic long-term survivors of childhood cancer treated with anthracyclines. Paediatric Blood Cancer 2009;52:631-6.

51. Cil T, Kaplan AM, Altintas A, et al. Use of N-terminal pro-brain natriuretic peptide to assess left ventricular function after adjuvant doxorubicin therapy in early breast cancer patients: a prospective series. Clin Drug Investig 2009;29:131-7.

52. Romano S, Fratini S, Ricevuto E, et al. Serial measurements of NT-proBNP are predictive of not-high-dose anthracycline cardiotoxicity in breast cancer patients. Br J Cancer 201 1; 105:1663-8.

53. Roziakova L, Bojtarova E, Mistrik M, et al. Abnormal cardio markers in leukaemia patients treated with allogeneic haematopoietic stem cell transplantation. Bratisl Lek Listy 20 I2; 1 1 3:159-62.

54. Sherief LM, Ahmad GK, Khalek EA, et al. Biomarkers and early detection of late onset anthracycline-induced cardiotoxicity in children. Haematol 2012; 17:151-6.

55. Lipshultz SE, Miller TL, Scully RE, et al. Changes in cardiac biomarkers during doxorubicin treatment of paediatric patients with high-risk acute lymphoblastic leukaemia: associations with long-term echocardiographic outcomes. J Clin Oncol 2012;30: 1042-9.

56. El Ghandour AH, El Sorady M, Azab S, et al. Human heart-type fatty acid-binding protein as an early diagnostic marker of doxorubicin cardiac toxicity. Haematol Rev. e6:I (2009)

57. Horacek JM, Tichy M, Pudil R, et al. Glycogen phosphorylase BB could be a new circulating biomarker for detection of anthracycline cardiotoxicity. Ann Oncol 2008; 19:1656-57.

58. Mercuro G, Cadeddu C, Piras A, et al. Early epirubicin-induced myocardial dysfunction revealed by serial tissue Doppler echocardiography: correlation with inflammatory and oxidative stress markers. Oncologist 2007; 1 2:1 124-33.

59. Cadeddu C, Piras A, Mantovani G, et al. Protective effects of the angiotensin II receptor blocker telmisartan on epirubicin-induced inflammation, oxidative stress and early ventricular impairment. Am Heart J 2010; 160:487.el-7.

60. Nuver J, Smit AJ, Sleijfer DT, et al. Microalbuminuria, decreased fibrinolysis and inflammation as early signs of atherosclerosis in long-term survivors of disseminated testicular cancer. Eur J Cancer 2004:40:70 I-6.

61. Vaughn DJ, Palmer SC, Carver JA, et al. Cardiovascular risk in long-term survivors of testicular cancer. Cancer 2008; 1 12:1949-53.

62. Altena R, Perik P, van Veldhuisen DJ, et al. Cardiovascular toxicity caused by cancer treatment: strategies for early detection. Lancet Oncol 2009; 10:391-99.
63. Wouters KA, Kremer LCM, Miller TL, et al. Protecting against anthracyclinesinduced myocardial damage: a review of the most promising strategies. $\mathrm{Br}$. Haematol 2005; 1 31:561-78.

64. Cardinale D, Colombo A, Cipolla CM. Prevention and treatment of cardiomyopathy and heart failure in patients receiving cancer chemotherapy. Curr Treat Options Cardiovasc Med 2008; 1 0:486-95

65. Spallarossa P, Garibaldi S, Altieri P, et al. Carvedilol prevents doxorubicin-induced free radicals release and apoptosis in cardiomyocytes in vitro. J Mol Cell Cardiol 2004:37:837-46.

66. Kalay N, Basar E, Ozdogru I, et al. Protective effects of carvedilol against anthracyclines-induced cardiomyopathy. J Am Coll Cardiol 2006;48:2258-62.

67. Van Dalen EC, Caron HN, Dickinson HO, et al. Cardioprotective interventions for cancer patients receiving anthracyclines. Cochrane Database Syst Rev 2011;6:CD003917.

68. Lipshultz SE, Rifai N, Dalton VM, et al. The effect of dexrazoxane on myocardia injury in doxorubicin-treated children with acute lymphoblastic leukaemia. N Eng J Med 2004;351:| 45-53.

69. Huh WW, Jaffe N, Durand JB, et al. Comparison of doxorubicin cardiotoxicity in paediatric sarcoma patients when given with dexrazoxane versus continuous infusion. Paediatric Haematol Oncol 2010;27:546-57.

70. Tebbi CK, London WB, Friedman D, et al. Dexrazoxane-associated risk for acute myeloid leukaemia/myelodysplastic syndrome and other secondary malignancies in paediatric Hodgkin's disease. J Clin Oncol 2007;25:493-500.

71. Barry EV, Vrooman LM, Dahlberg SE, et al. Absence of secondary malignant neoplasms in children with high-risk acute lymphoblastic leukaemia treated with dexrazoxane. J Clin Oncol 2008;26:I 106-II.

72. Nakamae H, Tsumura K, Terada Y, et al. Notable effects of angiotensin II receptor blocker, valsartan, on acute cardiotoxic changes after standard chemotherapy with cyclophosphamide, doxorubicin, vincristine and prednisolone. Cancer 2005; 104:2492-8

73. Ederhy S, Massard C, Dufaitre G, et al. Frequency and management of Troponin I elevation in patients treated with molecular targeted therapies in phase I trials. Invest New Drugs 2012;30:6 I I-5

74. Yoong G], Telli ML, Kao DP, et al. Left ventricular dysfunction in patients receiving cardiotoxic cancer therapies. J Am Coll 20 10;56:1644-50.

75. Ewer SM, Ewer MS. Cardiotoxicity profile of trastuzumab. Drug Safety 2008; 31:459-67.

76. Ewer MS, Vooletich MT, Durand JB, et al. Reversibility of trastuzumab-related cardiotoxicity. New insight based on clinical course and response to medical treatment. J Clin Oncol 2005,23:7820-6.

77. Tocchetti CG, Ragone G, Coppola C, et al. Detection, monitoring and management of trastuzumab-induced left ventricular dysfunction: an actual challenge. Eur J Heart Fail 2012; 14:130-137.

78. Telli ML, Hunt SA, Carlson RW, et al. Trastuzumab-related cardiotoxicity: calling into question the concept of reversibility. J Clin Oncol 2007;25:3525-33.

79. Cardinale D. Cardiac dysfunction after cancer treatment. In: Bonow RO, Mann DL, Zipes DP, Libby P (eds). Braunwald's Heart Disease E-dition. Chapter 90: Cardiovascular complication of cancer therapeutic agents. Philadelphia: Elservier Inc., 2012: 9th edition 